\title{
Design Optimization and Construction of the Thyratron/PFN Based Cost Model Modulator for the NLC
}

\author{
Ronald Koontz et al.
}

Presented at IEEE Particle Accelerator Conference (PAC 99), 3/29/99—4/2/99, New York, NY, USA 


\title{
DESIGN OPTIMIZATION AND CONSTRUCTION OF THE THYRATRON/PFN BASED COST MODEL MODULATOR FOR THE NLC*
}

\author{
Roland Koontz rfkap@slac.stanford.edu, Saul Gold, Anatoly Krasnykh, John Eichner, \\ Bin \#33 SLAC P. O. Box 4349, Stanford, CA 94309
}

\begin{abstract}
As design studies and various $R \& D$ efforts continue on Next Linear Collider (NLC) systems, much R\&D work is being done on X-Band klystron development, and development of pulse modulators to drive these X-Band klystrons. A workshop on this subject was held at SLAC in June of 1998, and a follow-up workshop is scheduled at SLAC June 23-25, 1999. At the 1998 workshop, several avenues of $R \& D$ were proposed using solid state switching, induction LINAC principles, high voltage hard tubes, and a few more esoteric ideas. An optimised version of the conventional thyratron-PFN-pulse transformer modulator for which there is extensive operating experience is also a strong candidate for use in the NLC. Such a modulator is currently under construction for base line demonstration purposes. The performance of this "Cost Model" modulator will be compared to other developing technologies. Important parameters including initial capital cost, operating maintenance- cost, reliability, maintainability, power efficiency, in addition to the usual operating parameters of pulse flatness, timing and pulse height jitter, etc. will be considered in the choice of a modulator design for the NLC. This paper updates the progress on this "Cost Model" modulator design and construction.
\end{abstract}

\section{INTRODUCTION}

The history of conventional modulator development for the NLC is covered in previous papers enumerated in the references. A brief description of the NLC concept is repeated here for reader's benefit. The NLC is a 1TEV, electron-positron linear collider, wherein two 500GEV linear accelerators are aimed at each other. Each LINAC is $10 \mathrm{~km}$ long, and operates at X-band, (11.424 GHz). Each LINAC employs over 3,000 75 MW klystrons, two klystrons per modulator. LINACS are divided into Sectors, each Sector containing 72 klystrons and 36 modulators in the Base Line configuration. An 8-pack induction modulator concept described in the Cassel paper in this conference could reduce the modulator count per sector to 9 , each modulator powering 8 klystrons. This is new technology, however, so at the present time, both conventional modulator and induction modulator developments are moving ahead in the NLC R\&D.

Efficiency, reliability and performance are major elements in the design requirements. SLAC's cost model modulator is based upon conventional technology and components that exist today, with the idea of optimising the components to meet the design criteria [2]. In the last year, various pulse discharge capacitors have been evaluated. These results indicate that at present, a conventional polypropylene design with attention to reducing series inductance is the best compromise where power transfer efficiency and reliability are prime considerations. Some work has been done by thyratron manufacturers to develop an appropriate thyratron having a 50,000-hour MTBF (mean time between failure). There is an effort to develop or co-ordinate development of a high voltage charging power supply with $95+\%$ efficiency. A solid state on-off switch is bcing considered as a replacement for the thyratron-PFN in a conventional modulator design. For a conventional modulator, a proposed physical size and layout has been developed which is being used in other areas of the NLC design efforts that interact with modulators.

Table 1 delineates the latest requirements for the klystron-modulator assembly. It is expected that these parameters can be achieved using current component technology.

Table 1 Baseline Modulator Requirements

\begin{tabular}{|l|l|}
\hline Parameter & Value \\
\hline Peak Klystron Voltage & $500 \mathrm{kV}$ \\
\hline Total Peak Current & $530 \mathrm{~A}$ \\
\hline Pulse Width(usable FT) & $1.5 \mu \mathrm{s}$ \\
\hline Pulse Top Flatness & $2 \%$ \\
\hline Pulse Top Ripple & $2 \%$ \\
\hline Pulse-pulse Ripple & $0.25 \%$ \\
\hline Pulse-pulse Jitter & $10 \mathrm{~ns}$ \\
\hline P.R.F. & $120 \mathrm{~Hz}$ \\
\hline Charging Voltage & $80 \mathrm{kV} \mathrm{max}$. \\
\hline Charging Supply Power. & $75 \mathrm{~kW}$ \\
\hline Charging Supply Efficiency. & $90 \%$ \\
\hline Overall Efficiency & $61.5 \%$ \\
\hline Reliability (MTBF) & $8,100 \mathrm{hr}$ \\
\hline
\end{tabular}

\section{ENERGY STORAGE CAPACITORS}

Pulse shape can be very dependent on the type of energy storage capacitor used. A fast rate of rise of current can be limited by the internal inductance of the capacitor itself, especially during a high current discharge. $\mathrm{R}$. Cassel and Saul Gold have tested film and ceramic capacitors for their internal inductance and found inductance of greater than $100 \mathrm{nHy}$ in most designs. A Sicond solid dielectric capacitor from Russia [2] was tested and found to have less than 30nHy of internal inductance, but at the expense of increased losses. After 
all of this testing, a standard polypropylene capacitor with special attention to end connections to minimise series inductance has been chosen for the "Cost Model" development.

\section{THYRATRON SWITCHES}

There is a long history of thyratron operation at SLAC. Presently, high power thyratrons have an average lifetime in excess of 15,000 hours [4]. A lifetime of 50,000 hours is desired for the NLC. Discussions with EEV, Litton and Triton, the three main thyratron manufacturers, have indicated that life increases with decreasing peak and average current. The $80 \mathrm{kV}$ charging voltage of the "Cost Model" modulator dictates a peak thyratron current at about 7500 amperes and an average current below 2 amperes. Three gap thyratrons are currently being developed by all three manufacturers to meet these requirements. The "Cost Model" modulator design will accept any of these three new thyratron models. In the next two years, samples of these thyratrons will be operated continuously in the SLAC LINAC and Klystron Test Lab to establish basic lifetime and reliability profiles.

\section{PULSE TRANSFORMERS}

Pulse transformer design, for the most part, is a mature technology, which can produce high reliability, relatively low loss voltage step-up to klystron cathode pulse potential. For most prior applications, energy transfer efficiency, and pulse rise time were not primary requirements of the transformer design. In the case of the NLC, these two parameters are of significant interest as they affect the operating cost of the two LINACs. Both Stangenes Industries and North Star Research have supplied SLAC with prototype NLC transformers. The testing results of these two units are given in [5]. Out of this initial testing, a new transformer design is emerging which will be used in the first version of the "Cost Model" modulator.

\section{PHYSICAL TANK LAYOUT}

The physical layout of the modulator is of critical importance to performance, reliability and maintainability and manufacturability. The sheer numbers of modulators required demands a layout, which affords ease of manufacturing and cost minimisation. We have built a full-scale partial mock-up of the modulator tank to help in this design effort, and have further modified it as the design was optimised. A photo of this mock-up is shown in the adjacent column.

The cross-section view of the modulator tank is shown in Fig 3. All components that are part of the pulse power delivery system are housed in a single tank, which also mounts the two $\mathrm{X}$ band klystrons. The two klystrons and

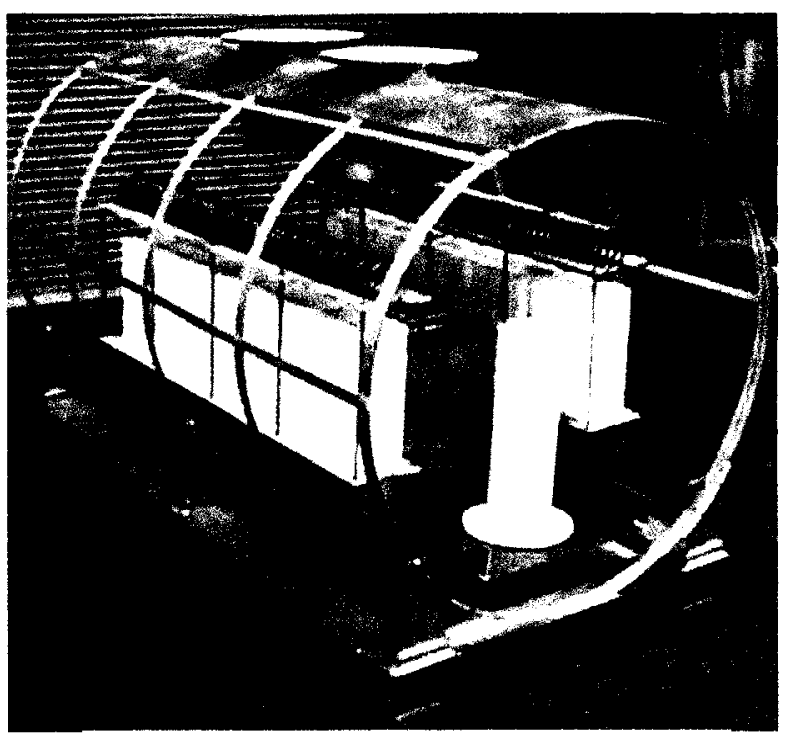

Fig.2 Modulator Tank Physical Mock-up

modulator tank assembly forms a single maintenance unit that will be removed to a repair facility when any of the modulator components, or the klystrons fail. This removes the need to handle oil in the Klystron Alcoves. One exception to this procedure may be the replacement of the thyratron if that unit requires more frequent replacement. The tank design is such that the thyratron can be plugged and unplugged through the oil expansion column on the top of the tank without removal of the oil.

A key to designing a fast rise time, electrically quiet modulator is to take into account, and minimise, all interconnecting impedances and stray capacitance that do not show up on the schematic diagram. The component layout in this tank design is based on minimising these loss-producing elements. Each high current path is mapped separately and isolated to its intended circuit via very low impedance connections, and ferrite isolated supports. Circuit elements that move up and down in voltage with the pulsing are designed to have minimum capacity to the grounded walls of the tank. Voltage holdoff margins are adequate for $500 \mathrm{kV}$ pulses, but excessive size and spacing which leads to inductive or capacitive losses are minimised.

Most of the components needed to build the first version of the "Cost Model" modulator are on hand. A detailed design of the tank assembly was completed and given to tank fabricators for costing. The resulting cost for a prototype unit was much higher than expected, so at this time, the design is being modified to reduce the fabrication cost. It is expected that an order for a reduced scope modulator tank will be placed in the next two months, and a "Cost Model" modulator will be in operation by the end of this fiscal year. 
Oil expansion reservoir \&

Thyratron removal port

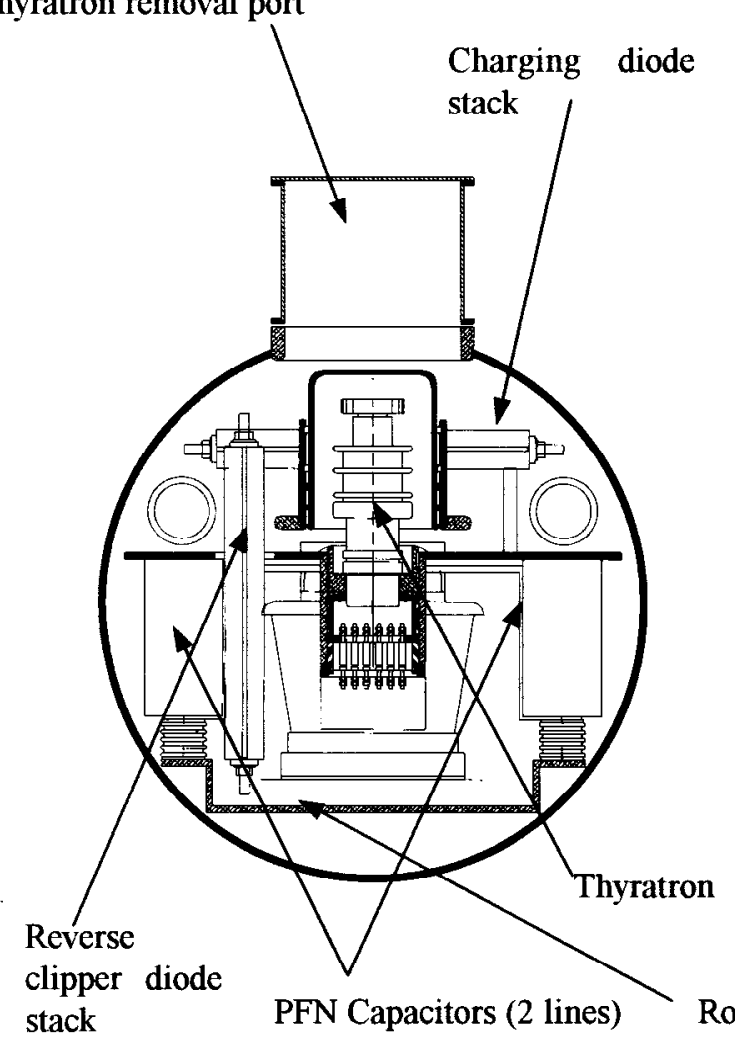

High voltage power

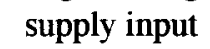

Two X band PPM 75 MW

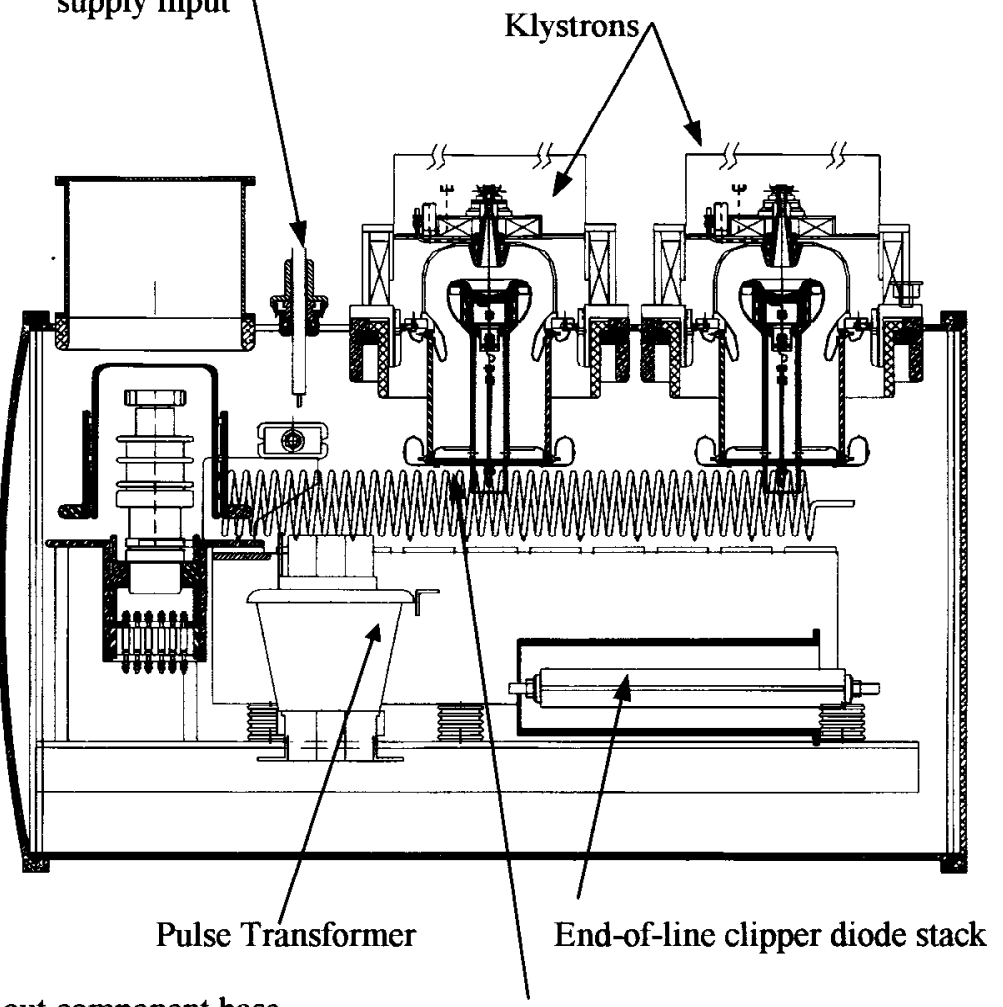

Mutually coupled PFN Inductors

Fig 3 Cross-section views of modulator components in tank

*Work supported by Department of Energy contract DE-AC03-76SF00515.

\section{References}

[1] The NLC design group: 'Zeroth-Order Design Report for the Next Linear Collider', SLAC-474, 1996.

[2] R. Koontz, M. Akemoto, S. Gold, A. Krasnykh, Z. Wilson: 'NLC Klystron Pulse Modulator R\&D at SLAC', Proceeding of 1997 Particle Accelerator Conference, Vancouver,B.C Canada, 1997.

[3] M. Akemoto, S. Gold: 'Optimization of a PFN with Mutual Coupling', to be published.

[4] D.B. Hicklin Jr.: 'An Updated History of the Thyratron Lifetimes at the Stanford Linear Accelerator Center', 23 International Modulator Symposium, Rancho Mirage, CA, 1998.

[5] S.L. Gold, JP. Eichner, R.F. Koontz, A. Krasnykh 'Developments in the NLC Modulator R\&D Program at SL $\Lambda C^{\prime} 23^{\text {rd }}$ Pulse Modulator Symposium 1998

[6] This paper can be obtained from SLAC Publications as SLAC-PUB-8073 